\title{
Strength of Effective Coulomb Interactions in Graphene and Graphite
}

\author{
T. O. Wehling, ${ }^{1}$ E. Şaşığlu, ${ }^{2}$ C. Friedrich, ${ }^{2}$ A. I. Lichtenstein, ${ }^{1}$ M. I. Katsnelson, ${ }^{3}$ and S. Blügel ${ }^{2}$ \\ ${ }^{1}$ Institut für Theoretische Physik, Universität Hamburg, D-20355 Hamburg, Germany \\ ${ }^{2}$ Peter Grünberg Institut and Institute for Advanced Simulation, Forschungszentrum Jülich and JARA, D-52425 Jülich, Germany \\ ${ }^{3}$ Radboud University Nijmegen, Institute for Molecules and Materials, NL-6525 AJ Nijmegen, The Netherlands
}

(Received 25 January 2011; published 8 June 2011)

To obtain an effective many-body model of graphene and related materials from first principles we calculate the partially screened frequency dependent Coulomb interaction. In graphene, the effective on-site (Hubbard) interaction is $U_{00}=9.3 \mathrm{eV}$ in close vicinity to the critical value separating conducting graphene from an insulating phase emphasizing the importance of nonlocal Coulomb terms. The nearestneighbor Coulomb interaction strength is computed to $U_{01}=5.5 \mathrm{eV}$. In the long-wavelength limit, we find the effective background dielectric constant of graphite to be $\epsilon=2.5$ in very good agreement with experiment.

DOI: 10.1103/PhysRevLett.106.236805

PACS numbers: 73.22.Pr, 71.45.Gm

The role of Coulomb interactions in graphene and related materials poses a long standing problem: experiments reported ferromagnetic ordering in nanographene [1], in disordered graphite samples [2], and at grain boundaries in highly oriented pyrolytic graphite (HOPG) [3]. Ferromagnetism in pristine graphene, however, has been excluded experimentally for temperatures down to $2 \mathrm{~K}$ [4]. Theoretically, the possibility of magnetism in defect free graphene has been predicted: an antiferromagnetic insulating ground state has been obtained for the local Coulomb interactions exceeding a critical value $U_{\mathrm{AFM}} \gtrsim$ $(4.5 \pm 0.5) t$ in quantum Monte Carlo (QMC) calculations [5-7] and $U_{\mathrm{AFM}} \gtrsim 2.2 t$ in Hartree-Fock theory [5,6], where $t \approx 2.8 \mathrm{eV}$ is the nearest-neighbor hopping parameter. A gapped spin liquid has been predicted for on-site repulsion between $U_{\mathrm{SL}}=3.5 t$ and $U_{\mathrm{AFM}}$ [8]. Sizable nonlocal Coulomb interactions can make the phase diagram even richer and lead to a competition between spin- and charge-density-wave phases $[9,10]$ or topologically nontrivial phases [11]. Doping of graphene might trigger further instabilities $[12,13]$. In pristine graphene, the Coulomb interaction remains long ranged and it is controversial whether this might lead to strongly correlated electronic phases like an insulator $[9,14]$ or whether graphene is rather weakly correlated. The local part of Coulomb interaction is also crucial for the theory of defect-induced magnetism in graphene [15].

The central issue in this discussion is the effective strength of the Coulomb interaction acting on the carbon $p_{z}$ electrons, which has only been estimated very roughly up to now [16]: The bare on-site Coulomb interaction in benzene obtained from atomic carbon $p_{z}$ orbitals was estimated to be $16.9 \mathrm{eV}$ [17]. For polyacetylene, an analysis of optical modulation spectroscopy experiments within weak coupling perturbation theory yielded an effective on-site Coulomb repulsion of $10 \mathrm{eV}[18,19]$. However, in this regime weak coupling perturbation theory might be inapplicable. For the long-wavelength limit, reflectance measurements of graphite [20] yielded a dielectric constant of $\epsilon=2.4$ due to screening by the high-energy $\sigma$ bands. This would correspond to an effective fine structure constant of $\alpha=\frac{e^{2}}{\epsilon \hbar v_{F}} \approx 0.9$ for bulk graphite, where $\hbar v_{F} \approx$ $5.8 \mathrm{eV} \AA$ is the Fermi velocity [16]. For graphene, recent inelastic x-ray scattering experiments [21] suggest a fully screened dielectric constant of $\epsilon \approx 15$ corresponding to a fine structure constant of $\alpha=0.14$. At the same time, firstprinciples $G W$ calculations [22] give $\epsilon \approx 4$, in agreement with the predictions of a simple Dirac model [16]. Recent experimental data on charge density dependence of the Fermi velocity [23] seem to be in agreement, rather, with the second value. So, up to now the strength of Coulomb interactions in graphene related materials has remained unclear and controversial — both theoretically and experimentally (for a review of correlation effects in graphene, see Ref. [24]).

In this Letter, we determine the Coulomb interaction strength in graphene and graphite within the constrained random phase approximation (cRPA) [25]. We obtain $a b$ initio effective Coulomb interaction parameters that should be used in a generalized Hubbard model of graphene or graphite (see cRPA values in Table I). We find that the on-site interactions in free standing graphene are weaker than $U_{\mathrm{AFM}}$ but close to the transition to the insulating spin-liquid phase at $U_{\mathrm{SL}}=3.5 t \approx 9.8 \mathrm{eV}$. Our calculations stress the importance of nonlocal Coulomb interactions in graphene. They put graphene in close proximity to two quantum phase transition lines and at the same time are possibly crucial for stabilizing a conducting state of freely suspended graphene. In the long-wavelength limit, we find bulk graphite having an effective background dielectric constant $\epsilon \approx 2.5$, in agreement with the experiments from Ref. [20]. For graphene in the long-wavelength limit $\epsilon$ is just one, as it should be for any two-dimensional system as will be explained below. 
TABLE I. On-site $\left(U_{00}^{A}, U_{00}^{B}\right)$, nearest-neighbor $\left(U_{01}\right)$, nextnearest-neighbor $\left(U_{02}^{A}, U_{02}^{B}\right)$, and third-nearest-neighbor $\left(U_{03}\right)$ (intralayer) Coulomb interaction parameters for freestanding graphene and graphite. In graphene $U_{00}^{A}=U_{00}^{B}$ and $U_{02}^{A}=U_{02}^{B}$ due to the sublattice symmetry. The bare and partially screened (cRPA) parameters are given. The cRPA parameters should be used in the effective Hamiltonian (1).

\begin{tabular}{lcccc}
\hline \hline & \multicolumn{2}{c}{ Graphene } & \multicolumn{2}{c}{ Graphite } \\
\hline & Bare & cRPA & Bare & cRPA \\
\hline$U_{00}^{A}$ or $B(\mathrm{eV})$ & 17.0 & 9.3 & $17.5,17.7$ & $8.0,8.1$ \\
$U_{01}(\mathrm{eV})$ & 8.5 & 5.5 & 8.6 & 3.9 \\
$U_{02}^{A}$ or $B(\mathrm{eV})$ & 5.4 & 4.1 & $5.4,5.4$ & $2.4,2.4$ \\
$U_{03}(\mathrm{eV})$ & 4.7 & 3.6 & 4.7 & 1.9 \\
\hline \hline
\end{tabular}

We start with constructing a generalized Hubbard model for the graphene $\pi$ bands,

$$
\begin{aligned}
\hat{H}_{0}= & -t \sum_{\langle\mathbf{i}, \mathbf{j}\rangle, \sigma} c_{\mathbf{i}, \sigma}^{\dagger} c_{\mathbf{j}, \sigma}-t^{\prime} \sum_{\langle\langle\mathbf{i}, \mathbf{j}\rangle, \sigma} c_{\mathbf{i}, \sigma}^{\dagger} c_{\mathbf{j}, \sigma}+U_{00} \sum_{\mathbf{i}} n_{\mathbf{i}, \uparrow} n_{\mathbf{i}, \downarrow} \\
& +\frac{1}{2} \sum_{\mathbf{i} \neq \mathbf{j}, \sigma, \sigma^{\prime}} U_{\mathbf{i j}} n_{\mathbf{i}, \sigma} n_{\mathbf{j}, \sigma^{\prime},}
\end{aligned}
$$

where $c_{\mathbf{i}, \sigma}$ annihilates an electron with spin $\sigma \in\{\uparrow, \downarrow\}$ at site $\mathbf{i}$ and $n_{\mathbf{i}, \sigma}=c_{\mathbf{i}, \sigma}^{\dagger} c_{\mathbf{i}, \sigma}$. The index $\mathbf{i}=(i, A$ or $B)$ labels the sublattice $(A, B)$ and the unit cell centered at position $R_{i}, U_{\mathrm{ij}}$ are the Coulomb interaction parameters. The nearest-neighbor hopping is known to be $t \approx 2.8 \mathrm{eV}$ $[16,26]$ and the next-to-nearest-neighbor hopping $t^{\prime}$ depends on details of how the tight-binding parameters are determined: $0.02 t \lesssim t^{\prime} \lesssim 0.2 t$.

To obtain all parameters entering the Hamiltonian (1) from first principles, we performed density functional theory (DFT) and cRPA calculations. The DFT calculations are carried out with the FLEUR code [27] using a generalized gradient approximation [28] for the exchangecorrelation energy functional. We use a linear momentum cutoff of $G_{\max }=4.5 \mathrm{bohr}^{-1}$ for the plane waves and an angular momentum cutoff of $l_{\max }=6$ in the muffin-tin spheres. The partially screened Coulomb matrix elements are calculated in the cRPA with the SPEX code $[29,30]$ using the mixed product basis $[29,31,32]$ with cutoff values $G_{\max }^{\prime}=4 \mathrm{bohr}^{-1}$ and $L_{\max }=4$.

The Hamiltonian (1) describes a system of $\mathrm{C} p_{z}$ electrons that interact via the effective interaction $U_{\mathrm{ij}}$, which incorporates the screening effects of all other electrons not contained in the Hamiltonian (1). The cRPA approach offers an efficient way to calculate this interaction [25], as the screening channels are individually accessible. The two-dimensional symmetry of graphene clearly separates the $\mathrm{C} p_{z}$ from other bands and, thus, enables an unequivocal elimination of the $\mathrm{C} p_{z}$ screening from the full RPA polarization function. Apart from the on-site term the resulting effective interaction yields the off-site, intraorbital, and interorbital terms as well as their frequency dependence.

The fully screened long-wavelength dielectric constants reported in Refs. [21-23] are different from the partially screened cRPA dielectric constants obtained, here, in that the former include also contributions to screening due to transitions between the graphene $\pi$ bands. Hence, using the dielectric constants from Refs. [21-23] in a generalized Hubbard model like Eq. (1) or in the context of investigations like Refs. $[9,14]$ would lead to double counting of screening terms arising from the $\pi$ electrons.

We ensure the accuracy of the model parameters being derived by carefully checking their dependence on the calculation procedure (the type of Wannier construction being used to define the $\mathrm{C} p_{z}$ orbitals) and convergence issues (Brillouin zone sampling and finite supercell height $h$ ) as we explain in the online supporting material [33]. We find that Wannier functions directly from the $\mathrm{C} p$ zrojections [34] and $16 \times 16 \times 1 k$ meshes for the $\mathrm{BZ}$ integration yield accurate Coulomb interaction parameters.

For graphene at its equilibrium lattice constant of $a_{0}=$ $2.47 \AA$, we obtain the Coulomb interaction parameters given in Table I. The on-site Coulomb repulsion $U_{00}^{A}$ or $B \approx$ $3.3 t$ is below $U_{\mathrm{AFM}} \approx(4.5 \pm 0.5) t$ [5-7] but very close to the critical value of $U_{\mathrm{SL}}=3.5 t$ separating the zero gap phase from a gapped spin-liquid one [8]. Comparing to the phase diagram reported in Ref. [10] our results show that the nearest-neighbor Coulomb interaction of $U_{01} \approx 2.0 t$ taken together with the local Coulomb interaction puts graphene in close proximity to, both, a spin-density wave and a charge-density-wave transition line.

The ratio of the kinetic energy given by $t$ to the Coulomb interaction can, e.g., be changed by applying strain. Upon expanding the graphene lattice the nearest-neighbor hopping decreases faster than the Coulomb interaction parameters [Fig. 1(a)]. An expansion of the lattice by a few percent leads to $U_{00}(a) / t(a)>3.5$, i.e., an increase of the ratio of local Coulomb interactions to the kinetic energy beyond the critical value of $U_{\mathrm{SL}} / t=3.5$. In this situation, the nonlocal Coulomb interaction effects can be crucial. It remains to be seen to which extent the long range nonlocal Coulomb interaction screens the on-site repulsion [35] and stabilizes the semimetallic phase or whether nonlocal Coulomb terms drive the system towards other strongly correlated possibly topologically nontrivial electronic phases as suggested in Refs. [10,11].

We now consider the Coulomb interaction in graphite and compare to graphene. In graphite, the two sublattices are not equivalent. We define the atoms of sublattice $A$ be to directly above each other in adjacent layers and sublattice $B$ as the atoms above hollow sites of the layer beneath. As Table I shows, the on-site interaction in graphene and graphite is qualitatively similar with very little difference between the two graphite sublattices. The ratio of bare to cRPA nearest-neighbor Coulomb interaction is 
(a)

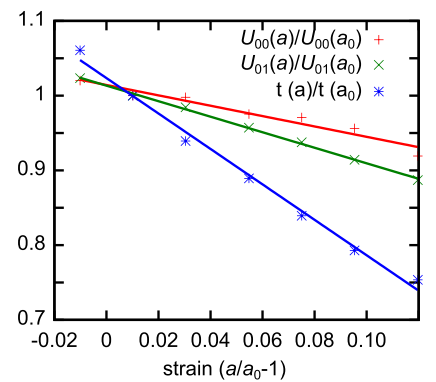

(b)

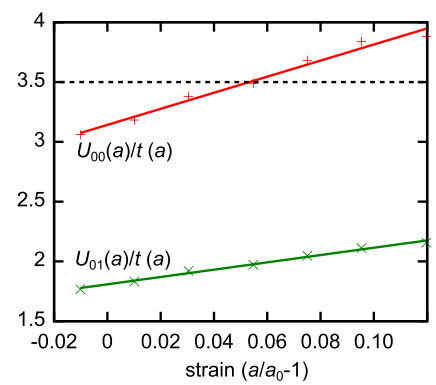

FIG. 1 (color online). Effect of lattice expansion on the strength of Coulomb interactions obtained with $h=21.2 \AA$. (a) On-site $U_{00}(a) / U_{00}\left(a_{0}\right)$ and nearest-neighbor $U_{01}(a) /$ $U_{01}\left(a_{0}\right)$ Coulomb interaction as well as nearest-neighbor hopping $t(a) / t\left(a_{0}\right)$ as a function of isotropic strain $\left(1-a / a_{0}\right) . a$ is the lattice constant. The parameters are given relative to their values at the equilibrium lattice constant $a_{0}=2.47 \AA$. The solid lines are linear fits serving as a guide to the eye. (b) Ratios of on-site $U_{00}(a) / t(a)$ and nearest-neighbor $U_{01}(a) / t(a)$ Coulomb interaction to the nearest-neighbor hopping. The dashed line indicates the phase boundary at $U_{\mathrm{SL}} / t=3.5$ separating the zero gap phase from a gapped spin-liquid one [8].

$U_{01}^{\text {bare }} / U_{01}^{\text {cRPA }}=1.6$ in graphene as compared to 2.2 in graphite. The nonlocal screening by the $\sigma$ bands is considerably more effective in graphite than in graphene.

This trend manifests clearly in the long-wavelength limit as can be seen from the Coulomb interaction in reciprocal space. To this end, we consider the Coulomb interaction matrix elements in terms of the Bloch transformed $\mathrm{C}$ $p_{z}$-Wannier functions, $\left|w_{n \mathbf{k}}\right\rangle$. We calculate the ratio of bare to cRPA screened interaction [36]

$$
\boldsymbol{\epsilon}(\mathbf{k})=\frac{\left\langle w_{n \mathbf{q}_{1}} w_{n \mathbf{q}_{2}+\mathbf{k}}\left|W^{\text {bare }}\right| w_{n \mathbf{q}_{1}+\mathbf{k}} w_{n \mathbf{q}_{2}}\right\rangle}{\left\langle w_{n \mathbf{q}_{1}} w_{n \mathbf{q}_{2}+\mathbf{k}}\left|W^{\mathrm{cRPA}}\right| w_{n \mathbf{q}_{1}+\mathbf{k}} w_{n \mathbf{q}_{2}}\right\rangle} .
$$

For graphene, our cRPA calculations (Fig. 2) yield $\epsilon(\mathbf{k}) \approx$ 2.4 for intermediate momentum transfer, $k=|\mathbf{k}| \gtrsim 1 \AA^{-1}$, and $\epsilon(\mathbf{k}) \rightarrow 1$ for $k \rightarrow 0$. The screening due to high-energy states in graphene becomes essentially negligible in the long-wavelength limit [37]. This is fundamentally different for graphite where $\epsilon\left(k_{\|}\right) \approx 2$ almost independently of the momentum transfer and $\epsilon(k=0) \approx 2.5$. Hence, graphite should be less correlated than graphene.

In the long-wavelength limit, the simplest model to address screening by high-energy bands in freestanding graphene is to consider a film of thickness $d$ and dielectric constant $\epsilon_{1}$. Transferring Ref. [38] to the geometry at hand [39] we obtain

$$
\begin{gathered}
\epsilon_{1}^{-1}(\mathbf{k})=\frac{1}{\epsilon_{1}} \frac{\epsilon_{1}+1+\left(\epsilon_{1}-1\right) e^{-k d}}{\epsilon_{1}+1-\left(\epsilon_{1}-1\right) e^{-k d}} \\
\stackrel{k \rightarrow 0}{\sim} 1+k d\left(\frac{1}{2 \epsilon_{1}}-\epsilon_{1}+1 / 2\right) .
\end{gathered}
$$
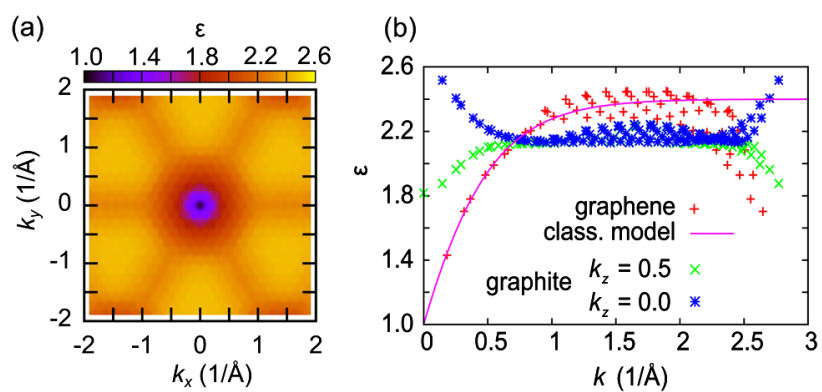

FIG. 2 (color online). Static cRPA dielectric functions $\epsilon(\mathbf{k})$ of graphene and graphite as function of (in-plane) momentum transfer $\mathbf{k}=\left(k_{x}, k_{y}\right)$. (a) Color coded (gray scale) $\epsilon(\mathbf{k})$ for graphene. The most pronounced effect is the decrease of $\epsilon(\mathbf{k})$ for $\mathbf{k} \rightarrow 0$. There is a small directional modulation at intermediate momentum transfer $k=|\mathbf{k}| \geqslant 1 \AA^{-1}$. (b) cRPA dielectric functions $\epsilon(k)$ of graphene and graphite as function of $k=|\mathbf{k}|$. Equation (3) fits well the background dielectric screening for freestanding graphene in the limit of $k \rightarrow 0$. For graphite, two values of the perpendicular momentum transfer are considered: $k_{z}=0$ and $k_{z}=0.5(2 \pi / c)$ with $c=3.3 \AA$ being the graphite interlayer spacing.

Our cRPA calculations confirm this expectation (see Fig. 2). Equation (3) turns out to describe the partially screened Coulomb interaction well for $k=|\mathbf{k}|<1 \AA^{-1}$ with $d=2.8 \AA$ and $\epsilon_{1}=2.4$ proving the applicability of this classical model at long wavelengths.

Integrating out the graphene $\sigma$ bands and other highenergy states leads to frequency dependent effective Coulomb matrix elements. For graphene and graphite, the effective Coulomb interaction is significantly frequency dependent above $\omega \gtrsim 5 \mathrm{eV}$ (Fig. 3). Within the energy range of the Dirac spectrum, however $(\sim 2 \mathrm{eV})$, the Coulomb interaction can be well considered in the static limit.

In conclusion, the strength of Coulomb interactions in graphene and graphite is accurately determined by firstprinciples calculations. The local Coulomb interaction in graphene is $U_{00}^{A}$ or $B=9.3 \mathrm{eV} \approx 3.3 t$, which is very close to

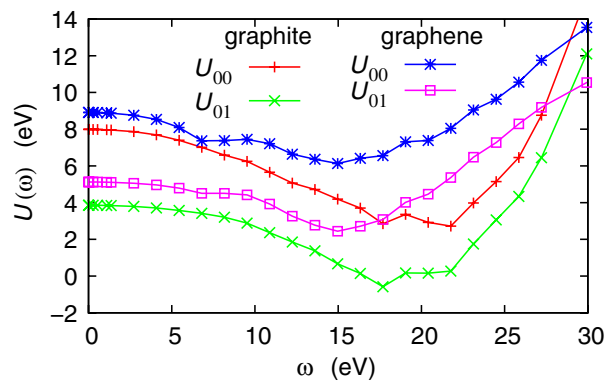

FIG. 3 (color online). Frequency dependence of the on-site and nearest-neighbor interaction obtained from cRPA for graphene $(h=21.2 \AA)$ and graphite. For graphite $U_{00}(\omega)=U_{00}^{A}(\omega)$ is shown, which is virtually the same as $U_{00}^{B}(\omega)$. $\mid U_{00}^{A}(\omega)-$ $U_{00}^{B}(\omega) \mid<0.15 \mathrm{eV}$ for $\omega<20 \mathrm{eV}$. 
the critical value $U_{\mathrm{SL}}=3.5 t$ for the transition to a gapped spin liquid. By straining graphene, the system can be driven across this critical value. Moreover, we find large nonlocal Coulomb interactions (e.g., $U_{01}=5.5 \mathrm{eV} \approx 2.0 t$ ). By means of a dielectric substrate below graphene the screening of the long range tails of the Coulomb interaction can be tuned, while the local Coulomb interaction terms are expected to be much less affected by the dielectric environment. Hence, also the ratio of local to nonlocal Coulomb interactions can be tuned. It remains to be seen which additional many-body instabilities might be triggered in this way or to which extent the conducting state of free standing graphene can be stabilized by nonlocal Coulomb terms. This issue deserves future attention. Very likely, our finding of large nonlocal Coulomb interaction $U_{01}$ in graphene generalizes to other two-dimensional materials. In narrow impurity bands or edge states of graphene, the Coulomb interaction might in any case present the dominating energy scale and, thus, trigger many-body instabilities including magnetism.

Financial support by the Deutsche Forschungsgemeinschaft through FOR-912, FOR-1346, SFB 668, SPP 1145, SPP 1459, and FOM (The Netherlands) is acknowledged. T. O. W. is grateful to FZ Jülich for hospitality during the visit, when parts of this work were conceived. One of us, S. B., thanks Achim Rosch for fruitful discussions.

[1] Y. Shibayama et al., Phys. Rev. Lett. 84, 1744 (2000); T. Enoki and K. Takai, Solid State Commun. 149, 1144 (2009).

[2] P. Esquinazi et al., Phys. Rev. B 66, 024429 (2002); Phys. Rev. Lett. 91, 227201 (2003).

[3] J. Cervenka, M. I. Katsnelson, and C. F. J. Flipse, Nature Phys. 5, 840 (2009).

[4] M. Sepioni et al., Phys. Rev. Lett. 105, 207205 (2010).

[5] S. Sorella and E. Tosatti, Europhys. Lett. 19, 699 (1992).

[6] L. M. Martelo et al., Z. Phys. B 103, 335 (1997).

[7] T. Paiva et al., Phys. Rev. B 72, 085123 (2005).

[8] Z. Y. Meng et al., Nature (London) 464, 847 (2010).

[9] I. F. Herbut, Phys. Rev. Lett. 97, 146401 (2006).

[10] C. Honerkamp, Phys. Rev. Lett. 100, 146404 (2008).

[11] S. Raghu et al., Phys. Rev. Lett. 100, 156401 (2008).

[12] N. M. R. Peres, M. A. N. Araújo, and D. Bozi, Phys. Rev. B 70, 195122 (2004).

[13] S. Pathak, V. B. Shenoy, and G. Baskaran, Phys. Rev. B 81, 085431 (2010).

[14] J.E. Drut and T. A. Lähde, Phys. Rev. Lett. 102, 026802 (2009).

[15] M.P. López-Sancho, F. de Juan, and M. A.H. Vozmediano, Phys. Rev. B 79, 075413 (2009).

[16] A. H. Castro Neto et al., Rev. Mod. Phys. 81, 109 (2009).
[17] R. G. Parr, D. P. Craig, and I. G. Ross, J. Chem. Phys. 18, 1561 (1950).

[18] Z. Vardeny and J. Tauc, Phys. Rev. Lett. 54, 1844 (1985).

[19] D. Baeriswyl, D. K. Campbell, and S. Mazumdar, Phys. Rev. Lett. 56, 1509 (1986).

[20] E. A. Taft and H. R. Philipp, Phys. Rev. 138, A197 (1965).

[21] J. P. Reed et al., Science 330, 805 (2010).

[22] M. van Schilfgaarde and M. I. Katsnelson, Phys. Rev. B 83, 081409 (2011).

[23] D. C. Elias et al., arXiv:1104.1396.

[24] V. N. Kotov et al., arXiv:1012.3484.

[25] F. Aryasetiawan et al., Phys. Rev. B 70, 195104 (2004); , 74, 125106 (2006).

[26] S. Reich et al., Phys. Rev. B 66, 035412 (2002).

[27] http://www.flapw.de.

[28] J. P. Perdew, K. Burke, and M. Ernzerhof, Phys. Rev. Lett. 77, 3865 (1996).

[29] C. Friedrich, A. Schindlmayr, and S. Blügel, Comput. Phys. Commun. 180, 347 (2009); C. Friedrich, S. Blügel, and A. Schindlmayr, Phys. Rev. B 81, 125102 (2010).

[30] E. Şaşığlu et al., Phys. Rev. B 81, 054434 (2010); E. Şaşığlu, C. Friedrich, and S. Blügel, ibid.83, 121101(R) (2011).

[31] F. Aryasetiawan and O. Gunnarsson, Phys. Rev. B 49, 16214 (1994)

[32] T. Kotani and M. van Schilfgaarde, Solid State Commun. 121, 461 (2002).

[33] See supplemental material at http://link.aps.org/ supplemental/10.1103/PhysRevLett.106.236805 for technical details for the cRPA calculations.

[34] W. Ku, H. Rosner, W. E. Pickett, and R. T. Scalettar, Phys. Rev. Lett. 89, 167204 (2002).

[35] R. Chitra and G. Kotliar, Phys. Rev. Lett. 84, 3678 (2000); P. Sun and G. Kotliar, Phys. Rev. B 66, 085120 (2002).

[36] Here, $\epsilon(\mathbf{k})$ is defined as the ratio of the bare and partially screened electron-electron interaction potentials with momentum transfer $\mathbf{k}$, i.e., $\mathbf{q}_{1} \rightarrow \mathbf{q}_{1}+\mathbf{k}$ and $\mathbf{q}_{2}+\mathbf{k} \rightarrow \mathbf{q}_{2}$, averaged over the momenta $\mathbf{q}_{1}$ and $\mathbf{q}_{2}$. The Wannier function index $n$ is chosen to correspond to atoms in sublattice $A$.

[37] The long-wavelength behavior of $\epsilon(k)$ determines the screening of the long range tails of the Coulomb interaction. $\epsilon(k) \rightarrow 1$ for $k \rightarrow 0$ corresponds to an unscreened $1 / r$ tail of the Coulomb interaction.

[38] A. Emelyanenko and L. Boinovich, J. Phys. Condens. Matter 20, 494227 (2008).

[39] Here, we consider a point charge in the middle of the film and evaluate the Coulomb potential in the middle of the film. Using the conventions and nomenclature of Ref. [38] our situation corresponds to $z=0, d=h / 2$, $\kappa=0, \epsilon_{2}=\epsilon_{3}=1$, and $\beta_{13}=\beta_{12}^{\prime}=\left(\epsilon_{1}-1\right) /\left(\epsilon_{1}+1\right)$. Then, Eq. (4) of Ref. [38] leads after division by the bare interaction $(q / \lambda)$ to $\epsilon(k=\lambda)$ as in our Eq. (3). 